\title{
Problemática de estudio e investigación de la adicción a las redes sociales online en jóvenes y adolescentes
}

\author{
Rubicelia Valencia-Ortiz \\ Gerente de Innovación y Transformación Digital en Macmillan Education-México \\ rubicelia.valencia@macmillaneducation.com | https://orcid.org/0000-0003-4656-5456 \\ Julio Cabero-Almenara \\ Catedrático de Didáctica y Organización Escolar de la Universidad de Sevilla \\ cabero@us.es | https://orcid.org/0000-0002-1133-6031 \\ Urtza Garay Ruiz \\ Profesora agregada de la Universidad del País Vasco \\ urtza.garay@ehu.eus | https://orcid.org/0000-0001-7298-9274
}

Bárbara Fernández Robles

Docente de la Facultad de Humanidades y Ciencias Sociales de la Universidad Isabel I bfernandezrobles@gmail.com | https://orcid.org/0000-0002-1760-392X

\section{Extracto}

\begin{abstract}
El uso excesivo de las redes sociales puede provocar efectos contraproducentes en los jóvenes: aislamiento social, distorsión entre el mundo real y virtual, falta de atención hacia otras actividades, depresión, ansiedad, problemas de salud y fracaso escolar. Ante esta perspectiva, es importante ofrecer información sobre las redes sociales desde una visión educativa. Con este artículo se pretende analizar el impacto que tienen en los jóvenes y estudiar algunos elementos que hay que controlar desde el ámbito educativo. Para ello, se realizó una revisión bibliográfica por diversas fuentes científicas, empleando un total de 116 publicaciones. Tras el análisis de estas fuentes científicas, se concluyó que las instituciones educativas, los docentes y los padres desempeñan un papel significativo para disminuir los desafíos que aparecen al emplear las redes sociales. Las instituciones educativas y los docentes deben ofrecer medios para desarrollar competencias digitales que permitan a los jóvenes desenvolverse de forma adecuada con ellas. Respecto a los padres, es necesario brindarles información para que guíen a sus hijos en la utilización de estas tecnologías.
\end{abstract}

Palabras clave: redes sociales; adicción; internet; papel del docente; educación de los padres.

Fecha de entrada: 05-06-2020 / Fecha de revisión: 29-07-2020 / Fecha de aceptación: 09-09-2020

Cómo citar: Valencia-Ortiz, R., Cabero-Almenara, J., Garay Ruiz, U. y Fernández Robles, B. (2021). Problemática de estudio e investigación de la adicción a las redes sociales online en jóvenes y adolescentes. Tecnología, Ciencia y Educación, 18, 99-125. 


\title{
Problems of study and investigation of addiction to online social networks in young people and adolescents
}

\author{
Rubicelia Valencia-Ortiz \\ Julio Cabero-Almenara \\ Urtza Garay Ruiz \\ Bárbara Fernández Robles
}

\section{Abstract}

Mobile telephony and social networks have revolutionized the ways of communicating and relating to ourselves today, being clearly reflected in the social relationships of young people and adolescents. The excessive use of social networks can cause counterproductive effects on the youngest: social isolation, distortion between the real and virtual world, lack of attention to other activities, depression, anxiety, health problems and school failure. Given this perspective, it is important to offer information on social networks from an educational perspective. This article aims to analyze the impact of social networks on young people and specify some elements that must be controlled from the educational field. To this end, a bibliographic review has been carried out by various scientific sources, using a total of 116 publications. After analyzing these scientific sources, we can conclude that educational institutions, teachers and parents have a great role to play in reducing the challenges that appear when using social networks. For their part, educational and teaching institutions must offer means to develop digital skills that allow young people to adequately deal with ICT and social networks. Regarding parents, it is necessary to offer them information to guide their children in the use of these technologies.

Keywords: social media; addiction; internet; teacher role; parent education.

Citation: Valencia-Ortiz, R., Cabero-Almenara, J., Garay Ruiz, U. and Fernández Robles, B. (2021). Problems of study and investigation of addiction to online social networks in young people and adolescents. Tecnologia, Ciencia y Educación, 18, 99-125. 


\section{Sumario}

1. Introducción

2. Metodología

3. La adicción a las redes sociales: su conceptualización y diagnóstico

4. La adicción a las redes sociales por jóvenes y adolescentes: sus problemáticas

5. Una mirada a las acciones formativas

6. A manera de conclusión

Referencias bibliográficas 


\section{Introducción}

Internet se ha convertido en la tecnología ante la cual gira la sociedad de la información y ha sido, además, la que se ha extendido más velozmente en la historia de la humanidad. Como señala Oppenheimer (2018), solo necesitó siete años para extenderse desde EE. UU. al resto del mundo. En España, de acuerdo con el Observatorio Nacional de las Telecomunicaciones y Sociedad de la Información (ONTSI, 2019), en el año 2018, internet, a través de banda ancha, estaba presente en el $86,10 \%$ de los hogares, teniendo en los últimos años una tendencia ascendente.

Hoy no nos imaginaríamos el mundo sin su existencia y, posiblemente, el confinamiento que algunos países han decretado para sus ciudadanos por la COVID-19 habría sido menos llevadero sin la red, así como también habría sido imposible continuar con diferentes empresas sin el teletrabajo. Por ello se reclama la necesidad de unas adecuadas competencias digitales en la ciudadanía. En este sentido, el Consejo Europeo recomienda una «mejora de las capacidades básicas, especialmente en temas digitales y de tecnología de la información, como prioridad fundamental para convertir a la Unión en la economía basada en el conocimiento más competitiva y dinámica del mundo» (Comisión de la Unión Europea, 2001, p. 4). Competencias establecidas en nuestro contexto europeo a través de diferentes marcos competenciales europeos destinados a distintos colectivos: DigComp (ciudadanía), DigCompOrg (instituciones educativas) y DigCompEu (profesorado) (Cabero y Palacios, 2020; Carretero et al., 2017; Kampylis et al., 2015; Pérez-Escoda et al., 2019; Redecker y Punie, 2017).

Pero, paralelamente a esta presencia, también nos encontramos con dos tecnologías que se han extendido fuertemente durante los últimos años: la telefonía móvil y las redes sociales online (RSO). Por lo que se refiere a la primera, y específicamente entre el colectivo de jóvenes y adolescentes, su presencia y utilización ha proliferado enormemente (García y Fernández, 2016; Gértrudix et al., 2017).

Paralelamente a la presencia de internet, nos encontramos con dos tecnologías que se han extendido fuertemente durante los últimos años. Hablamos de la telefonía móvil y de las RSO

De acuerdo con el informe elaborado por la Fundación Orange (Orange, 2018), en España, el 45,90\% de los padres indican que han adquirido el primer teléfono móvil para sus hijos cuando estos tenían 14 años. La mayoría de los jóvenes y adolescentes (93,20 \%) lo utilizan para conectarse a internet, estando el 85,10\% de ellos apuntados como mínimo a una RSO (Manca y Ranieri, 2016; Tang et al., 2016). Como han señalado Peñalva y Napal 
(2019), «el alumnado que dispone de teléfono móvil va aumentando de manera progresiva con la edad y alcanza un $70 \%$ en el curso quinto y un $85 \%$ en el curso sexto» (p. 60).

La United Nations Educational, Scientific and Cultural Organization (UNESCO, 2013) resaltaba que para 2017 la mitad de la población de los países en desarrollo tendría como mínimo una conexión activa a un teléfono. Teniendo en cuenta que este auge aumenta con los años, nos podemos imaginar que su volumen sea hoy mayor.

Posiblemente, por la importancia y presencia que los dispositivos móviles están teniendo en nuestra sociedad (Gértrudix et al., 2017; Orange, 2018; Vázquez y Sevillano-García, 2018), las RSO se están convirtiendo en uno de los medios predilectos de los jóvenes y adolescentes para solicitar información y establecer comunicación e interacción con sus pares (Gordo et al., 2018). Como señalan García y Fernández (2016), «ningún medio de

Las RSO se están convirtiendo en uno de los medios predilectos de los jóvenes y adolescentes para solicitar información y establecer comunicación e interacción con sus amigos y compañeros comunicación ni situación ocurrida con anterioridad en la historia de la humanidad ha sido capaz de concentrar en tan "poco" espacio (una pequeña pantalla) a tal cantidad de personas» (p. 3).

Recientemente, Rubio (2019) señalaba que los jóvenes entre 14 y 24 años prefieren usar las aplicaciones de mensajería para comunicarse con familiares y amigos más que el medio telefónico. También resaltaba el uso diario de diferentes RSO por parte de los jóvenes.

El fuerte uso y abuso de la utilización de estos dos recursos tecnológicos ha llevado a diferentes autores a señalar que se da una fuerte adicción ante ellos (Mora et al., 2015). Este hecho lleva a considerarlo como un problema de salud pública y, por tanto, debe abordarse por diferentes instituciones, entre ellas la educativa (Malak et al., 2017).

Las RSO se han convertido en una de las herramientas más utilizadas para la comunicación y el intercambio de información entre las personas en diferentes sectores (ocio, negocio, formación, etc.). Este uso es reflejado en el Estudio Anual de Redes Sociales de IAB Spain (https:// iabspain.es/), indicando que el $85,5 \%$ de los internautas entre 16 y 65 años utilizan las RSO.

Las RSO tienen una serie de características específicas que las hacen seductoras para los jóvenes (Orange, 2018). Entre estas cualidades encontramos las siguientes (Fuentes et al., 2015; Musial y Kazienko, 2013):

Las RSO tienen una serie de características específicas que las hacen seductoras para los jóvenes

- Ausencia de contacto físico entre sus participantes.

- Favorecen la transformación de la personalidad del individuo en el mundo real y virtual. 
- Posibilitan la relación social para las personas que en la vida real presentan limitaciones.

- Posibilidad de poder comunicarse al mismo tiempo y de manera simultánea con un gran volumen de personas.

- Facilidad técnica para romper o suspender las relaciones y los contactos.

Lo más preocupante de las RSO es que, por sus características de anonimato, de fácil y rápido acceso, de desinhibición y de falta de contacto físico, pueden desencadenar diversos efectos perjudiciales (Fuentes et al. , 2015; Griffiths, 1995). $Y$ estos son los motivos que han influido para que se establezca una línea de investigación dirigida al estudio de la «adicción a las redes sociales online», que ha sido considerada como un tipo particular de adicción a internet (Sahin, 2018).

\author{
Lo más preocupante de las RSO \\ es que, por sus características \\ de anonimato, de fácil y rápido \\ acceso, de desinhibición y de \\ falta de contacto físico, pueden \\ desencadenar diversos efectos \\ perjudiciales (Fuentes et al., 2015; \\ Griffiths, 1995)
}

\section{Metodología}

Con el presente trabajo se pretendía analizar el impacto que tienen las redes sociales en los más jóvenes y especificar elementos que hay que controlar desde el ámbito educativo para integrarlas adecuadamente. Para tal fin, se realizó una revisión bibliográfica por diversas fuentes científicas relacionadas con el tema de estudio. Entre estas fuentes podemos destacar International Journal of Mental Health and Addiction, Journal of Information, Communication and Ethics in Society, Computers in Human Behavior, Píxel-Bit. Revista de Medios y Educación, Revista Complutense de Educación, Adicciones, Revista Española de Drogodependencia, Psychiatry Research, EDMETIC. Revista de Educación Mediática y $T I C$, etc.

Para la búsqueda y selección de la información se tuvieron en cuenta diversas palabras que se combinaron por pares o tríos con ayuda del conector «y»: «redes sociales», «redes sociales y educación», «internet y jóvenes», «internet y desafíos», «redes sociales y desafíos» y «educación y tecnología». Para crear estas combinaciones se han concretado previamente algunas palabras relacionadas con el tema de estudio: «redes sociales», «internet», «jóvenes», «educación», «desafíos» y «tecnología».

En total se han seleccionado 116 publicaciones, dando prioridad a documentos científicos de los últimos 10 años. Por este motivo, la mayoría de publicaciones empleadas corresponden al periodo 2010-2020, exceptuando tres publicaciones que son de años anteriores. En la figura 1 se puede apreciar el número de publicaciones utilizadas por periodo de tiempo. 
Figura 1. Distribución de publicaciones seleccionadas por año de publicación

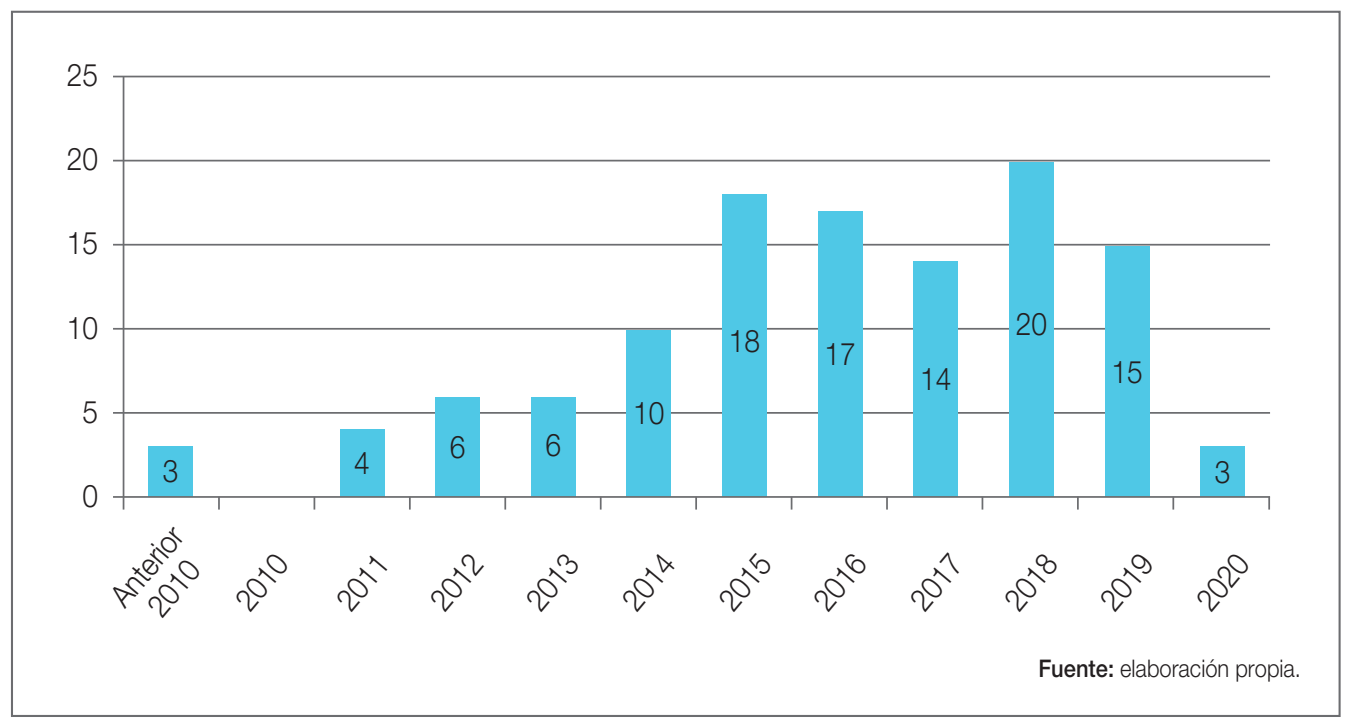

Para seleccionar las publicaciones se revisaron previamente los títulos y resúmenes con la finalidad de comprobar el grado de adecuación al tema de estudio. Tras seleccionar las fuentes se revisaron los documentos completos y se organizaron por bloques temáticos con el fin de facilitar la creación del marco teórico. Algunos de los bloques temáticos creados fueron "Concepto de red social», "Diagnóstico de adicción», "Desafíos de las redes sociales», «Responsabilidad del ámbito educativo en el uso de las redes sociales», «Instrumentos de diagnóstico», «Uso de internet TIC»y «Papel de los padres en el uso de internet».

\section{La adicción a las redes sociales: su conceptualización y diag- nóstico}

Vamos a comenzar señalando que el término «adicción» ha estado asociado al consumo de estupefacientes y sustancias químicas (Basteiro et al., 2013; Echeberúa, 2012). El Manual diagnóstico y estadístico de los trastornos mentales de la American Psychiatric Association (2016) especifica que la «adicción» es un abuso y dependencia de cualquier tipo de sustancias psicoactivas.

Esta idea inicial ha ido variando con el tiempo, pues diferentes autores señalan que el desarrollo de un proceso adictivo puede producirse sin la mediación de compuestos químicos. Como señalan Watters et al. (2013), por «adicción» podríamos entender un uso compulsivo y excesivo de una sustancia o actividad que provoca un deterioro en el funcionamiento diario de la persona y hace que se convierta en una conducta adictiva. Hecho que se puede 
observar en el trastorno producido por los juegos de internet, que, debido a los problemas que ha ocasionado, se ha incorporado como diagnóstico preliminar en la versión quinta del DSM-5 (American Psychiatric Association, 2016).

De todas formas, no todos los autores están de acuerdo en considerar que el uso abusivo de las RSO pueda considerarse como una conducta aditiva, ya que, a veces, se confunde la dependencia a una tecnología con la adicción a una conducta. Según estos autores sería más adecuado distinguir las auténticas adicciones de los excesos y de los problemas pasajeros de la exposición abusiva e intensa a una tecnología (Carbonell, 2014; Carbonell y Oberst, 2015; Kuss et al., 2013; Pontes et al., 2015).

Por otra parte, encontramos un grupo de autores (Arnavu et al., 2018; Delgado, 2019; Jasso et al., 2017; Sahin, 2018) que no consideran la adicción a las RSO de forma aislada, sino que la asocian a la adicción a los teléfonos móviles e internet.

Es de destacar que, respecto a la adicción a las RSO, nos encontramos con dos problemas. En primer lugar, la falta de acuerdo en torno a la consideración de la adicción a las tecnologías como un problema aditivo. No obstante, lo que sí es cierto es que se ha desarrollado una corriente de investigación que se inició con un estudio de la adicción a internet (Echeberúa, 2012; Fernández-Villa et al., 2015; Young, 2015), lo que llevó a la identificación de una serie de conductas en las personas que abusaban del uso temporal de esta tecnología (Chóliz y Marco, 2012):

- Tolerancia: cada vez se necesita estar más tiempo conectado.

- Abstinencia: el malestar que aparece cuando se interrumpe la conexión.

- El medio se utiliza más de lo pretendido inicialmente.

- Deseo por dejar de usar internet, sin poder dejarlo.

- Empleo excesivo de tiempo en actividades relacionadas con internet.

- Dejar de hacer otras actividades para poder usar más internet.

- Utilización de internet a pesar de saber que nos está perjudicando.

Y, en segundo lugar, la falta de concreción respecto al tiempo que debe invertir una persona para que pueda ser considerada como adicta a las RSO. De todas formas, lo que sí va aportando la investigación es que la gran utilización de las RSO repercute en diferentes conductas de las personas, como dificultades de flexibilidad cognitiva (Dong et al., 2014), problemas para la toma de decisiones (D’Hondt et al., 2015), aumento del nivel de angustia (Wegmann et al., 2015), olvido de la realización de ciertas actividades (Chóliz y Marco, 2012), baja autoestima (Herrera et al., 2010), dificultades en el desarrollo de la memoria de trabajo (Dong et al., 2012), conflictos en la concentración (Rücker et al., 2015) o problemas familiares (Fernández-Villa et al., 2015). 
Los aspectos señalados repercuten en un aislamiento social y generan la distorsión del mundo real, síntomas de abstinencia, limitación del sueño y la no realización de otras actividades, como las académicas (Echeberúa, 2012). También se ha sugerido, por motivo de su abuso, una relación lineal positiva entre el «neuroticismo», la extraversión, la depresión (Basteiro et al., 2013) y la confusión entre el mundo real y virtual (Llamas y Pagador, 2014).

A la hora de referirnos a su diagnóstico, lo primero que hay que señalar, de acuerdo con la propuesta realizada por Van Rooij y Prause (2014), es que nos encontramos con tres modelos para ello: el modelo de los componentes de Griffiths (2005); el modelo generado a partir del trabajo de Young (1998), que está unido a los indicadores del DSM-5 para la adicción a sustancias; y el de Tao et al. (2010), que parte del estudio de las características clínicas de pacientes que fueron diagnosticados como adictos a internet y tratados en ese sentido.

Por lo general, para su diagnóstico, los instrumentos utilizados se ubican dentro de los denominados «test de adicción a las redes sociales» (TARS), que suelen ser instrumentos de autopercepción y que están construidos en formato tipo Likert. Pocos son los construidos fuera de este formato, como el elaborado por Basteiro et al. (2013), que tiene una construcción escala dicotómica. En el cuadro 1, se presentan algunas dimensiones de estos instrumentos y sus autores.

Cuadro 1. Dimensiones de instrumentos y autores

Ocupación.

Modificación del estado de ánimo.

Tutgun-Ünal y Deniz (2015).

Conflicto.

Preocupación.

Tolerancia.

Retiro.

Desplazamiento.

Escape.

Van den Eijnden et al. (2016).

Problemas.

Decepción.

Desplazamiento.

Conflicto.

Pérdida de control.

Tolerancia.

Sanz et al. (2017).

Abstinencia que su utilización provoca. 


\begin{tabular}{l|l}
\multicolumn{1}{c|}{ Dimensiones } & Autores \\
\hline & \\
Abuso. & Chóliz y Marco (2012). \\
Obsesión y perturbación. & \\
Abstinencia y pérdida de control. & Schou et al. (2012). \\
Saliencia. & \\
Modificación del estado de ánimo. & \\
Tolerancia. & \\
Retiro. & \\
Conflicto. & \\
Recaída. & Sahin (2018). \\
Tolerancia virtual. \\
Comunicación virtual. \\
Adicción a los medios sociales. \\
Satisfacción/tolerancia.
\end{tabular}

\section{Conflicto.}

Síndrome de abstinencia.

Recaída y restablecimiento.

Turel y Serenko (2012).

Saliencia.

Retiro y problemas sociales.

Gestión del tiempo y rendimiento.

Al-Menayes (2015).

Sustitución de la realidad.

Persistencia.

Aburrimiento.

Uso excesivo.

Orosz et al. (2016).

Autoexpresión.

Uso compulsivo.

Abstinencia.

Consecuencia negativa.

Alteración del estado de ánimo.

Saliencia.

Liu y Ma (2018).

Tolerancia.

Recaída.

Preferencia por la interacción social en línea.

Uso continuo. 
Obsesión por las RSO.

Falta de control personal en el uso de las RSO.

Escurra y Salas (2014).

Uso excesivo de las RSO.

Satisfacción.

Problema.

Información.

Valencia y Castaño (2019).

Necesidad de estar conectado.

Fuente: elaboración propia.

Los instrumentos mayoritariamente utilizados son posiblemente una de las limitaciones de los resultados obtenidos en las investigaciones, puesto que se apoyan en la técnica del autoinforme de la información por parte del propio sujeto; técnica que conlleva problemas de subjetividad.

Dado el volumen de investigaciones realizadas sobre la adicción a las RSO se han publicado diferentes metaanálisis para estudiar y evaluar algunas de las escalas elaboradas (Castro y Moral, 2017; Sigerson y Cheng, 2018).

\section{La adicción a las redes sociales por jóvenes y adolescentes: sus problemáticas}

La utilización abusiva de las RSO, sobre todo por jóvenes y adolescentes, exige una llamada de atención por parte de la sociedad en general y de las instituciones educativas en particular.

Tal y como se ha puesto de manifiesto, el uso abusivo de internet y las RSO genera diferentes tipos de conductas y trastornos negativos para las personas: aislamiento social (Echeberúa, 2012; García y Tirado, 2018; Yu et al., 2016), distorsión entre el mundo real y digital (Llamas y Pagador, 2014), privación de sueño (Gordo et al., 2018; Xanidis y Brignell, 2018), desinterés por la realización de otras actividades (Li et al., 2016), depresión (Lozano-Blasco y Cortés-Pascual, 2020), problemas de relación con los familiares (Fernández-Villa et al., 2015), baja autoestima (Lee y Cheung, 2014), depresión (Boonvisudhi y Kuladee, 2017), problemas fisiológicos y de ansiedad como consecuencia de su abstinencia (Koyuncu et al., 2014; Kuss y Griffiths, 2017), fracaso escolar como consecuencia de la disminución del rendimiento académico (Afroz, 2016; Blachnio et al., 2016; Cañón et al., 2016; Malo et al., 2018) o problemas de salud (Li et al., 2016). 
También desde la biología se ha apuntado que la presencia de la persona de forma constante y abusiva en las RSO tiene consecuencias negativas. Desde esta perspectiva, el uso abusivo produce cambios en la conectividad neuronal, en la estructura y en el funcionamiento del cerebro (He et al., 2017; Se-Hoon et al., 2016).

Para una serie de autores (Carr, 2011, 2014; Watson, 2011), la fuerte exposición por parte de las personas a internet y a las tecnologías que se desenvuelven alrededor de la red trae repercusiones cognitivas: tendencia a la multitarea y, por tanto, a pasar de forma rápida de una tarea a otra; necesidad de inmediatez en las acciones y resultados; y preferencia por lo audiovisual frente a lo impreso. Tales características están repercutiendo en la disminución de la capacidad de atención, en la concentración y en la dificultad de movilizar un pensamiento profundo (Watson, 2011).

Esta situación lleva a Carr $(2011,2014)$ y a Watson (2011) a indicar que internet está cambiando no solo los comportamientos y las costumbres de las personas, sino también su forma de pensar.

No se puede olvidar que uno de los grandes problemas que se está produciendo en el uso de las RSO en la actualidad es el denominado «ciberbullying» o «ciberacoso». Esta forma de acoso supone la utilización de distintas tecnologías digitales con la pretensión de acosar psicológicamente a otras personas. Acoso que por lo general se da entre personas menores y entre adolescentes de la misma edad y que suelen pertenecer a idéntico contexto social (González y Prendes, 2018; Vizcaíno-Verdú et al., 2020). En el Informe Pisa 2018 se señalan como características que diferencian el «ciberacoso» del «acoso tradicional» las siguientes:

\begin{abstract}
El amparo en el posible anonimato, la suplantación, la relativa falta de miedo de los acosadores a ser identificados, la falta de supervisión y la sensación de las víctimas de que pueden ser intimidadas/acosadas en cualquier momento (Ministerio de Educación y Formación Profesional, 2019, p. 153).
\end{abstract}

Como se señala también en el citado informe, cuanto más tiempo se invierte en las RSO, más posibilidades hay de sufrir dicha conducta, así como que estudiantes con altos niveles de competencia digital presenten menos posibilidades de sufrir acoso. Igualmente, poseer un compromiso cívico se asocia negativamente con el acoso online y positivamente con comportamientos saludables en las RSO (Ministerio de Educación y Formación Profesional, 2019).

Como se ha comentado, uno de los grandes problemas a los que nos enfrentamos es que, por lo general, la adicción a las RSO se da mayoritariamente en jóvenes y adolescentes (Ahmadi, 2014; Castro y Moral, 2017; Chóliz y Marco, 2012; Gordo et al., 2018; Özdemir et al., 2014;). Esto, si se tiene presente que están en una fase de maduración cognitiva y emocional, lo hace más alarmante y resulta necesario que se le preste atención desde un punto de vista educativo (Kuss y Griffiths, 2017). Lo más preocupante es que, por lo general, cuando se les ha preguntado a los estudiantes si se consideraban adictos a las RSO por el 
número de horas que invertían en ellas, sus respuestas han sido mayoritariamente negativas (Marín et al., 2015; Kuss y Griffiths, 2017; Marín et al., 2019).

Relacionado con la institución escolar, una de sus consecuencias es la correlación negativa y significativa entre el aumento de horas invertidas en las RSO y el incremento del fracaso académico (Afroz, 2016; Blachnio et al., 2016; Cañón et al., 2016; Malo et al., 2018; Marín et al., 2019). Los estudiantes que invierten más horas en las RSO se sienten por lo general menos interesados por las actividades académicas y tienden a abandonarlas para disponer de más tiempo en la red. Normalmente, también son estudiantes que muestran menos interés por la realización de actividades extraescolares (Wang et al., 2013).

Aunque como encontraron Kim et al. (2017), no es solamente el tiempo invertido, sino también el uso que se hace de internet y de las RSO. De esta forma, un rendimiento académico alto se asocia con el uso de internet durante largas horas con fines académicos, sin embargo, muchas horas invertidas en actividades de ocio y lúdicas se asocia negativamente con el rendimiento académico. Al mismo tiempo, dedicar muchas horas a acciones de ocio estaba relacionado con la baja motivación hacia el estudio y con poca capacidad de autorregulación y autoeficacia hacia el aprendizaje (Marín et al., 2019; Reed y Reay, 2015).

La situación se complica, puesto que los estudiantes que suelen tener una fuerte adicción a las RSO tienden a relacionarse con compañeros que presentan también esta dependencia (Echeburúa y Requesens, 2012), con lo cual autorrefuerzan su propia conducta. Son, al mismo tiempo, estudiantes que presentan pobres relaciones sociales y suelen tender hacia el aislamiento social (Echeburúa y Requesens, 2012).
Los estudiantes que suelen tener una fuerte adicción a las RSO tienden a relacionarse con compañeros que presentan también esta dependencia, con lo cual autorrefuerzan su propia conducta adictiva

Últimamente se está potenciando la investigación entre el uso de las TIC y la comunicación desde una perspectiva de género, ya que una de las brechas digitales que se ha identificado es la de género (Cabero y Ruiz-Palmero, 2015; Yeganehfar et al., 2018).

Las investigaciones que se han realizado en esta línea aportan dos grandes orientaciones:

- Existen diferencias significativas en función del género de los estudiantes en la interacción que establecen con las RSO. Los hombres presentan mayor grado de adicción a las RSO que las mujeres (Beyens et al., 2016; Durán y Guerra, 2015; Golpe et al., 2017; Gordo et al., 2018; Lee, 2015; Turel et al.,
Existen diferencias significativas en función del género de los estudiantes en la interacción que establecen con las RSO. Los hombres presentan mayor grado de adicción a las RSO que las mujeres 
2014). Pocas son las investigaciones en las cuales se ha detectado una mayor adicción y un uso problemático de las RSO en las mujeres que en los hombres (Heo et al., 2014; Marín et al. , 2019; Marino et al., 2018) o que no existieran diferencias significativas entre ambos colectivos (Syahputra et al., 2019).

- Los hombres y las mujeres tienden hacia usos diferentes de las RSO. Así, Mazman y Usluel (2011) reportaron que los hombres y las mujeres hacen un uso diferente de Facebook. Estos autores indican que las mujeres lo utilizan para establecer contactos con sus amistades y los hombres para ampliar su círculo de amistades. Diferentes estudios (Durán y Guerra, 2015; Golpe et al., 2017; Mayor et al., 2019; Tejada et al. , 2019) detectaron que el uso que hacen las chicas y los chicos de internet y las RSO es diferente. Por lo general, las chicas las utilizan de forma más activa: llevan a cabo más trabajos académicos con ellas, buscan más información académica y realizan más actividades formativas.

\section{Una mirada a las acciones formativas}

Teniendo en cuenta los problemas que ocasiona el uso abusivo de las RSO, es imprescindible que las instituciones educativas ofrezcan respuestas para evitar o reducir daños que puedan repercutir notablemente en el futuro sociolaboral y personal del sujeto. Ya en el Informe Delors (UNESCO, 1996) se indicaba que la educación debería perseguir cuatro pilares básicos: aprender a conocer, aprender a hacer, aprender a vivir juntos y con los demás y aprender a ser. Posiblemente, la problemática que se está analizando se sitúa en los dos últimos tipos de aprendizaje, puesto que se requiere una formación para saber manejarnos en unos contextos de comunicación diferentes a los presenciales tradicionales y para controlar estas nuevas herramientas de comunicación y utilizarlas desde una perspectiva humanística y enriquecedora de la persona.

No podemos negar que los niños y adolescentes viven en un entorno mediático como nunca había ocurrido en la historia de la humanidad. Es más, como se ha comentado, para nuestros jóvenes sería incluso inimaginable una vida sin la interacción con ellos. Por tanto, es importante analizar y reflexionar sobre la influencia del mundo digital en la configuración de su personalidad, así como abordar medidas para que adquieran la competencia digital suficiente para saber desenvolverse en ellas de una forma segura. Esta reflexión urge, pues cada vez hay más jóvenes que acceden a dispositivos tecnológicos que les permiten la conexión a las RSO y a internet desde edades muy tempranas.

Lo comentado lleva a señalar que la situación debe abordarse desde diferentes perspectivas y actores: las instituciones educativas, los docentes y los padres. Todos ellos deben adoptar medidas de forma coordinada para el abordaje de la situación.

Por lo que se refiere a las instituciones educativas y a los profesores, se deben adoptar medidas para que los niños y jóvenes adquieran la suficiente competencia digital para que 
sepan desenvolverse de forma positiva en y con estas tecnologías. Si se revisan diferentes marcos competenciales, como, por ejemplo, el del Marco Europeo de Competencias Digitales para la Ciudadanía (DigComp -dentro del área «Seguridad en la Red» están las competencias «Protección de Datos Personales y Privacidad» y «Protección de la Salud y del Bienestar»-), se encuentran múltiples referencias claramente relacionadas con lo que estamos comentando (Carretero et al., 2017).

La adquisición de esta competencia repercutirá en que los estudiantes sean hábiles en el manejo ético y seguro de las RSO. De esta manera, lograrán una «dieta digital» que les enseñará a conectar y desconectar los dispositivos, a responder mensajes e interaccionar en la red en momentos específicos y a no estar constantemente atentos a lo que se produce en estos dispositivos. Sería alcanzar la resistencia íntima de la que habla Esquirol (2015), para referirnos, con este término, «no tanto a las dificultades que el mundo pone a nuestra disposición como a la fortaleza que podemos tener y levantar ante los procesos de desintegración y corrosión que provienen del entorno e incluso de nosotros mismos» (p. 10).

Por lo que se refiere a los padres, uno de los problemas con el que nos enfrentamos es la adopción de medidas para potenciar un uso seguro de las RSO por los hijos, teniendo en cuenta el bajo conocimiento que los padres suelen tener respecto a su funcionamiento y problemática de utilización (Moreno et al., 2017). Formación que es muy relevante, pues, como reconocen los estudiantes cuando son conscientes de su grado de adicción, es a ellos a los que tienden a pedir ayuda (Plaza, 2018). Por otra parte, diferentes estudios han puesto de manifiesto que la adicción de los jóvenes a las RSO está relacionada con el
Por lo que se refiere a los padres, uno de los problemas con el que nos enfrentamos es la adopción de medidas para potenciar un uso seguro de las RSO por los hijos, teniendo en cuenta el bajo conocimiento que los progenitores suelen tener respecto a su funcionamiento y problemática de utilización nivel de formación de sus padres (Gordo et al., 2018; Lai y Kwan, 2017). Al mismo tiempo se debe reconocer que los padres muestran un verdadero interés tanto para que sus hijos tengan una buena formación a la hora de hacer un uso responsable y positivo de las RSO como para el suyo propio (Dans et al., 2019).

En el caso de los padres, se hace necesario que estos conozcan los posibles problemas y las consecuencias que puede tener el hecho de que sus hijos abusen de las RSO e internet. Como han señalado Martínez-Piñeiro et al. (2018), las familias desempeñan un papel relevante en la construcción de la competencia digital de los jóvenes y adolescentes, y su clase social determina patrones de conductas diferentes respecto a la permisividad de sus hijos hacia las tecnologías. Al respecto, el colectivo de docentes reclama que exista en los domicilios un horario restringido, lugares específicos y tiempos para su uso. Por otra parte, como determinaron García et al. (2019) en su investigación, el tiempo que los padres dedican a sus hijos influye en su mayor autoestima, en la mejora de la capacidad de relacionarse socialmente, en la potenciación de sus actitudes prosociales y en otros aspectos. 
Esta situación lleva a proponer la necesidad de establecer acciones formativas en las instituciones para que los alumnos adquieran la competencia que les permita hacer un uso correcto de las RSO y que propicie beneficios en la persona. Para tal fin, es importante el control del tiempo que el sujeto dedica a ellas, puesto que esto puede mejorar su nivel de satisfacción con la vida y, al mismo tiempo, ofrecer beneficios psicológicos para la persona (Rad y Ahmadi, 2018). Por ello, es interesante comprender los diferentes modelos que intentan explicar la adicción a las RSO (Kuss y Griffiths, 2017):

- Cognitivo-conductual. Sugiere que la adicción a las RSO es consecuencia de cogniciones no adaptativas.

- Habilidades sociales. La preferencia del sujeto por un modelo de comunicación cara a cara como consecuencia, entre otros motivos, de la timidez del individuo.

- Sociocognitivo. Indica que el uso excesivo de las RSO es consecuencia de las expectativas de resultados positivos, de la autoeficacia y de la autorregulación limitada de internet.

Una propuesta interesante es el modelo de «administración del conocimiento personal» (personal knowledge management [PKM]) propuesto por Fong et al. (2016), que persigue capacitar a los estudiantes para un uso positivo de las RSO en una serie de dimensiones: búsqueda, evaluación, organización, colaboración, análisis, presentación y protección.

Debido a la importancia que está alcanzando la concienciación del uso correcto de las redes sociales e internet en adolescentes, son diversas las iniciativas que se han desarrollado para evitar o reducir posibles efectos contraproducentes por el uso inadecuado de estas tecnologías. En el cuadro 2 se reflejan algunas iniciativas que se han llevado a cabo para concienciar a educadores, futuros profesionales de la educación, padres, madres y alumnos. No obstante, debemos resaltar que las experiencias e iniciativas en este campo se encuentran todavía en un estado incipiente.

Cuadro 2. Iniciativas para concienciar sobre el uso de internet y las redes sociales

\section{Destinatarios}

Docentes.

\section{Contenido de la iniciativa}

Acción formativa destinada a docentes para obtener competencias en el uso de la Web 2.0 y de las TIC, centrando la atención en las redes sociales en uno de los talleres.
Autores

Acceso a la iniciativa

González et al. (2011). https://www.redalyc.org/ pdf/2010/201022649003.pdf 
Destinatarios

\section{Contenido de la iniciativa}

\section{Autores}

Acceso a la iniciativa
Futuros profesiona- Propuesta didáctica sobre les de la educación. el uso de las redes sociales destinada a estudiantes de pedagogía.
Gutiérrez y Ballesteros (2013). https://idus.us.es/handle/ $11441 / 59104$
Gobierno de Extremadura.
Alumnado, familia y Guía para el buen uso de las profesionales de la enseñanza.

Padres, madres y educadores.

\author{
fesionales.
}

Guía para padres y educadores sobre el uso seguro de internet, móviles y videojuegos.
Labrador et al. (2015).

https://iespereda.es/ampa/ docs/Guia.para.el.buen.uso. educativo.de.las.TIC.pdf

https://iespereda.es/ampa/ docs/Guia.para.padres.y. educadores.sobre.el.uso.se guro.de.Internet, . moviles. y.videojuegos.pdf

Padres y madres. Guía de formación TIC para padres y madres de adolescentes. En el capítulo 3 se centra la atención en las redes sociales.

Padres y madres. Guía para uso seguro y resdiación parental.
Junta de Andalucía.

https://www.andaluciaesdigi tal.es/internet-seguro/educarpara-proteger https://www.is4k.es/sites/ default/files/contenidos/he rramientas/is4k_guia_media cion_parental_internet.pdf ponsable de internet por los menores. Itinerario de me-

Ministerio de Industria, Energía y Turismo del Gobierno de España/ Instituto Nacional de Ciberseguridad/Oficina de Seguridad del Internauta.

Junta de Andalucía.

Guía de formación TIC para padres y madres de menores de 3 a 11 años.

Padres y madres. Guía para padres y madres para ayudar a sus hijos a usar correctamente la televisión, el teléfono móvil, los videojuegos e internet.
Gobierno de Navarra/ Consejo Audiovisual de Navarra.
Padres y madres. 


\begin{tabular}{|c|c|c|c|}
\hline Destinatarios & Contenido de la iniciativa & Autores & Acceso a la iniciativa \\
\hline \multicolumn{4}{|l|}{4} \\
\hline Alumnos. & $\begin{array}{l}\text { Programa formativo desti- } \\
\text { nado a discentes de } 6 .^{\circ} \text { de } \\
\text { educación primaria y a alum- } \\
\text { nos de educación secunda- } \\
\text { ria obligatoria (ESO) sobre el } \\
\text { uso seguro y responsable de } \\
\text { internet. }\end{array}$ & $\begin{array}{l}\text { Instituto Nacional de Se- } \\
\text { guridad (https://www. } \\
\text { incibe.es///nternet Se- } \\
\text { gura for Kids (https:// } \\
\text { www.is4k.es/). }\end{array}$ & $\begin{array}{l}\text { https://www.is4k.es/sites/ } \\
\text { default/files/contenidos/ma } \\
\text { teriales/privacidad_ud.pdf }\end{array}$ \\
\hline Alumnos. & $\begin{array}{l}\text { Guía pedagógica para tra- } \\
\text { bajar la educación en las } \\
\text { nuevas tecnologías: uso res- } \\
\text { ponsable, preventivo y edu- } \\
\text { cativo. }\end{array}$ & $\begin{array}{l}\text { Servicio Central del Go- } \\
\text { bierno Vasco. }\end{array}$ & $\begin{array}{l}\text { https://www.euskadi.eus/ } \\
\text { contenidos/informacion/egon } \\
\text { line_unitatedidaktikoak/es_def/ } \\
\text { adjuntos/egonline_cast.pdf }\end{array}$ \\
\hline Alumnos. & $\begin{array}{l}\text { Unidad didáctica «Internet se- } \\
\text { guro para alumnos». }\end{array}$ & $\begin{array}{l}\text { Dirección Provincial de } \\
\text { Educación Salamanca. }\end{array}$ & $\begin{array}{l}\text { http://ceipjoaquindiaz.cen } \\
\text { tros.educa.jcyl.es/sitio/upload/ } \\
\text { Taller_alumnos___Unidad_Di } \\
\text { dactica_Internet_seguro_ } \\
\text { para_alumnos_SA.pdf }\end{array}$ \\
\hline
\end{tabular}

Fuente: elaboración propia.

\section{A manera de conclusión}

El uso que los jóvenes y adolescentes hacen de las RSO es una temática que debe ser abordada por la institución educativa con preocupación, pues en la red se enfrentan a una serie de riesgos que van desde la gestión de su contenido y huella digital, hasta la privacidad de la información que exponen y su imagen (imagen social y personal).

Un uso abusivo de las RSO y de internet por parte de los jóvenes y adolescentes puede traer como consecuencia la aparición de problemas psicológicos, afectivos, de salud y académicos. Por ello se hace necesario que desde las instituciones educativas se lleven a cabo acciones en diferentes direcciones y sobre distintos actores: estudiantes, profesores y padres de familia.

Por lo que se refiere a los profesores, se hace necesario que adquieran competencias digitales centradas en la temática del uso seguro de las RSO por parte de los estudiantes. Formación que debe centrarse en diferentes aspectos: la problemática del ciberacoso, la inseguridad del uso abusivo de las RSO por parte de los estudiantes y los problemas que esto 
les pueda acarrear, y, por supuesto, saber organizar acciones formativas dirigidas a estos aspectos. Esta formación no debe realizarse exclusivamente para los profesores que estén en ejercicio, sino también ha de incorporarse en los planes de formación de los futuros maestros (infantil y primaria) y profesores (secundaria y bachillerato).

Por parte de los docentes, la formación debe centrarse en la adquisición de elementos que propicien en sus estudiantes la reflexión sobre los efectos negativos del abuso de usar las RSO, en la orientación al estudiante a la hora de que estos adquieran conductas para autorregular su uso de las RSO y competencias para alcanzar una dieta digital, en promover un uso crítico de las RSO, en adquirir estrategias para la puesta en acción de actividades formativas, en organizar actividades dirigidas a los padres y madres, etc. También cabe hacernos una pregunta: ¿los docentes tienen competencias digitales suficientes en esta materia como para emprender acciones formativas e informativas para sus estudiantes y los padres? En este aspecto pueden surgir una serie de dudas (Mateus y Hernández, 2019).

Las actividades formativas deben centrarse también en los padres. Actividades formativas que, reconozcámoslo, son demandadas por los propios padres, ya que ellos son conscientes de los problemas que conlleva, tanto para sus hijos como para la propia familia, el uso abusivo de las RSO (Dans et al., 2019; Moreno et al., 2017). En el caso de los padres, las acciones formativas deben dirigirse a adquirir competencias a la hora de discriminar conductas que les hagan suponer que sus hijos pueden tener un posible problema aditivo tecnológico (inversión de demasiado tiempo en el consumo de tecnología digital, aislamiento progresivo de las relaciones familiares, cambios de conducta al prohibirse su uso, etc.). También, en la adquisición de acciones que permitan que sus hijos puedan ir abandonando el uso excesivo de las RSO y de las tecnologías digitales (establecimiento de una dieta digital, explorar alternativas con los hijos de inversión del tiempo, potenciación de la realización de otras actividades, etc.).

Diferentes instituciones autonómicas están llevando a cabo distintas acciones formativas que pueden servir de guía al docente para afrontar su acción con estudiantes y padres. Este es el caso de las acciones formativas de la Comunidad de Madrid (Franco, 2016) o de las elaboradas por el Ayuntamiento de Valencia (Sánchez et al., 2018).

Aunque la investigación en adicción a las RSO está aportando una serie de datos fundamentales, se echan en falta estudios que se centren en el diseño, la producción y la evaluación de planes formativos destinados a superar la adicción a las RSO: contenidos que hay que impartir, modalidades de puesta en acción, material didáctico que se debe emplear, etc. Hasta el momento, los resultados aportados son fundamentalmente de carácter descriptivo respecto al número de horas invertidas, actividades realizadas o tipo de red utilizada.

Para finalizar, hay que destacar que los jóvenes actuales crecen en una realidad en la que se encuentran desconectados de sus semejantes, pero, a la vez, más conectados que nunca, lo que puede provocar efectos contraproducentes en su desarrollo (Goleman, 2013). 


\section{Referencias bibliográficas}

Afroz, N. (2016). Internet addiction and subjective well-being of university students. Indian Journal of Health and Wellbeing, 7(8), 787-794.

Ahmadi, K. (2014). Internet addiction among Iranian adolescents: a nationwide study. Acta Medica Iranica, 52(6), 467.

Al-Menayes, J. (2015). Psychometric properties and validation of the arabic social media addiction scale. Hindawi Publishing Corporation Journal of Addiction, ID 291743. http:// dx.doi.org/10.1155/2015/291743

American Psychiatric Association. (2016). Diagnostic and Statistical Manual of Mental Disorders: DSM-5.

Arnavut, A., Nuri, C. y Direktor, C. (2018). Examination of the relationship between phone usage and smartphone addiction based on certain variables. Anales de Psicología, 34(3), 446-450. https://doi.org/10.6018/ana lesps.34.3.321351

Ávila, D. L., Pardo, E. L. y Muñoz, M. L. (2019). Escala de adicción al internet de Lima (EAIL): análisis psicométrico. Revista Iberoamericana de Psicología, 11(3), 103-111. https://doi. org/10.33881/2027-1786.rip.11309

Basteiro, J., Robles, A., Juarros, J. y Pedrosa, I. (2013). Adicción a las redes sociales: creación y validación de un instrumento de medida. Revista de Investigación y Divulgación en Psicología y Logopedia, 3(1), 2-8.

Beyens, I., Frison, E. y Eggermont, S. (2016). «I don't want to miss a thing»: adolescents' fear of missing out and its relationship to adolescents' social needs, Facebook use, and Facebook related stress. Computers in Human Behavior, 64, 1-8. https://doi.org/10. 1016/j.chb.2016.05.083

Błachnio, A., Przepiorka, A. y Pantic, I. (2016). Association between Facebook addiction, self-esteem and life satisfaction: a cross-sec- tional study. Computers in Human Behavior, 55, 701-705. https://doi.org/10.1016/j.chb. 2015.10.026

Boonvisudhi, T. y Kuladee, S. (2017). Association between internet addiction and depression in Thai medical students at Faculty of Medicine, Ramathibodi Hospital. PLOS ONE, 12(3), e0174209. https://doi.org/10.1371/journal. pone.0174209

Cabero, J. y Palacios, A. (2020). Marco Europeo de Competencia Digital Docente «DigComp Edu» y cuestionario «DigCompEdu Check-In». EDMETIC. Revista de Educación Mediática y T/C, 9(1), 213-234. https://doi.org/10.21071/ edmetic.v9i1.12462

Cabero, J. y Ruiz-Palmero, J. (2018). Las tecnologías de la información y la comunicación para la inclusión: reformulando la brecha digital. International Journal of Educational Research and Innovation, 9, 16-30. https:// www.upo.es/revistas/index.php/IJERI/ article/view/2665/2222

Cañón, S. C., Castaño, J. J., Hoyos, D. C., Jaramillo, J. C., Leal, D. R., Rincón, R. y Urueña, L. S. (2016). Uso de internet y su relación con la salud en estudiantes universitarios de la ciudad de Manizales (Caldas-Colombia), 2015-2016. Archivos de Medicina (Manizales), 16(2), 312-325. https://doi.org/10.3 0554/archmed.16.2.1735.2016

Carbonell, X. (2014). ¿Qué son las adicciones tecnológicas? Internet, MMORPG y redes sociales. En X. Carbonell (Eds.), Adicciones tecnológicas: qué son y cómo tratarlas (pp. 53-85). Síntesis.

Carbonell, X. y Oberst, U. (2015). Las redes sociales en línea no son adictivas. Aloma. Revista de Psicologia, Ciències de l'Educació i de l'Esport, 33(2), 13-19.

Carr, N. (2011). Superficiales: ¿qué está haciendo internet con nuestras mentes? Taurus. 
Carr, N. (2014). Atrapados: cómo las máquinas se apoderan de nuestras vidas. Taurus.

Carretero, S., Vuorikari, R. y Punie, Y. (2017). DigComp 2.1: The Digital Competence Framework for Citizens With Eight Proficiency Levels and Examples of Use. Publication Office of the European Union. https://doi.org/10. $2760 / 38842$

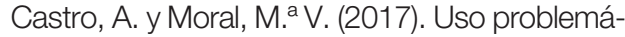
tico de redes sociales 2.0 en nativos digitales: análisis bibliográfico. Health and Addictions/ Salud y Drogas, 17(1), 73-85. https://doi.org/ 10.21134/haaj.v17i1.284

Chóliz, M. y Marco, C. (2012). Adicción a internet y redes sociales: tratamiento psicológico. Alianza.

Comisión de la Unión Europea. (2011). Proyecto de programa de trabajo detallado para el seguimiento del informe sobre los objetivos concretos de los sistemas de educación y formación. https://www.uv.es/oce/web\%20 castellano/base\%20datos/educ\%20y\%20 formac\%20europa.pdf

D'Hondt, F., Billieux, J. y Maurage, P. (2015). Electrophysiological correlates of problematic internet use: critical review and perspectives for future research. Neuroscience and Biobehavioral Reviews, 59, 64-82. https:// doi.org/10.1016/j.neubiorev.2015.10.005

Dans, I., González-Sanmamed, M. y MuñozCarril, P. C. (2019). Redes sociales, adolescencia y familia: desafíos y oportunidades. Publicaciones, 49(2), 117-132. https://doi. org/10.30827/publicaciones.v49i2.8527

Delgado, P. (28 de junio de 2019). La adicción a los celulares preocupa a estudiantes y docentes. Observatorio de Innovación Educativa.

Dong, G., Devito, E., Du, X. y Cui, Z. (2012). Impaired inhibitory control in «internet addiction disorder». Psychiatry Research, 203(2-3), 153-158. https://doi.org/10.1016/j.pscychre sns.2012.02.001
Dong, G., Lin, X., Zhou, H. y Lu, Q. (2014). Cognitive flexibility in internet addicts: $\mathrm{fMRl}$ evidence from difficultto-easy and easy-to-difficult switching situations. Addictive Behaviors, 39, 677-683. https://doi.org/10.1016/j.add beh.2013.11.028

Durán, M. y Guerra, J. (2015). Usos y tendencias adictivas de una muestra de estudiantes universitarios españoles a la red social Tuenti: la actitud positiva hacia la presencia de la madre en la red como factor protector. Anales de Psicología, 31(1), 260-267. https://doi. org/10.6018/analesps.31.1.158301

Echeburúa, E. (2012). Factores de riesgo y factores de protección en la adicción a las nuevas tecnologías y redes sociales en jóvenes y adolescentes. Revista Española de Drogodependencia, 37(4), 435-447. https://www.ae sed.com/descargas/revistas/v37n4_5.pdf

Echeburúa, E. y Requesens, A. (2012). Adicción a las redes sociales y nuevas tecnologías en niños y adolescentes: guía para educadores. Ediciones Pirámide.

Eijnden, R. van den, Lemmens, J. y Valkenburg, P. (2016). The social media disorder scale. Computers in Human Behavior, 61, 478-487.

Escurra, M. y Salas, E. (2014). Construcción y validación del cuestionario de adicción a redes sociales (ARS). LIBERABIT, 20(1), 73-91. https://www.redalyc.org/pdf/686/68 631260007.pdf

Esquirol, J. M. (2015). Laresistenciaíntima. Acantilado.

Fernández-Villa, T., Aguacil, J., Almaraz, A., Cancela, J. M. ${ }^{a}$, Delgado-Rodríguez, M., GarcíaMartín. M., Jiménez-Mejías, E., Llorca, J., Molina, A. J., Ortiz, R., Valero-Juan, L. F. y Martín. V. (2015). Uso problemático de internet en estudiantes universitarios: factores asociados y diferencias de género. Adicciones, 27(4), 265-275. https://doi.org/10.208 82/adicciones. 751 
Fong, B., Lo, M. F. y Ng, A. (2016). A theoretical model to integrate PKM with Kolb's learning model for mitigating risks from exhaustive internet exposures. Electronic Journal of Knowledge Management, 14(3), 166-176.

Franco, J. (Coord.). (2016). TICmenoRes-Programa para la prevención de riesgos y el uso adecuado de las TRIC. Comunidad de Madrid.

Fuentes, J. L., Esteban, F. y Caro, C. (2015). Vivir en internet: retos y reflexiones para la educación. Síntesis.

García. M. ${ }^{a}$ C. y Fernández. C. (Coords.). (2016). Si lo vives, lo compartes. Cómo se comunican los jóvenes en un mundo digital. Fundación Telefónica-Ariel.

García, J. y Juanes, J. A. (2013). El cerebro y las TIC. Teoría de la Educación, 14(2), 42-84.

García, A. y Tirado, R. (2018). Digital media behavior of school students: abusive use of the internet. Journal of New, 7(2),140-147. https://doi.org/10.7821/naer.2018.7.284

García, J. M., Lacalle, M.a, Valbuena, C. M. ${ }^{a}$ y Polaino, A. (2019). Relaciones familiares y tiempo compartido y su efecto en el bienestar de los adolescentes. Revista Complutense de Educación, 30 (3), 895-915. https:// doi.org/10.5209/rced.59562

Gértrudix, M., Borges, E. y García, F. (2017). Vidas registradas. Redes sociales y jóvenes en la era algorítmica, Telos, 107, 62-70.

Goleman, D. (1997). La inteligencia emocional. Kairos.

Golpe, S., Gómez, P., Kim, S., Braña, T. y Boubeta, A. (2017). Diferencias de sexo en el uso de internet en adolescentes españoles. Behavioral Psychology/Psicología Conductual, 25(1), 129-146.

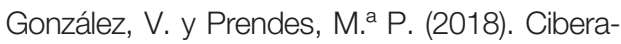
cosadores: un estudio cuantitativo con estudiantes de secundaria. Píxel-Bit. Revista de
Medios y Educación, 53, 137-144. https:// doi.org/10.12795/pixelbit.2018.i53.09

González, F., Carretero, M., Escudero, J. y Arraz, O. (2011). Niños 2.0, una experiencia formativa en actitudes y valores para el profesorado ante la Web 2.0 y TIC. TESI, 12(4), 25-38.

Gordo, Á., García, A., Rivera, J. de y Díaz-Catalán, C. (2018). Jóvenes en la encrucijada digital: itinerarios de socialización y desigualdades en los entornos digitales. Morata.

Griffiths, M. (1995). Technological addictions. Clinical Psychology Forum, 95, 14-19.

Gutiérrez, N. y Ballesteros, M. Á. (2013). Una propuesta didáctica sobre el uso de las redes sociales en el contexto universitario. III Jornadas de Innovación Docente: Respuesta en Tiempos de Incertidumbre. Sevilla, España: Universidad de Sevilla. Facultad de Ciencias de la Educación. https://idus.us.es/ handle/11441/59104

He, Q., Turel, O. y Bechara, A. (2017). Brain anatomy alterations associated with social networking site (SNS) addiction. Scientific Reports, 7. https://doi.org/10.1038/srep45 064

Heo, J., Oh, J., Subramanian, S. V., Kim, Y. y Kawachi, I. (2014). Addictive internet use among Korean adolescents: a national survey. PLOS ONE, 9(2), e87819. https://doi. org/10.1371/journal.pone.0087819

Herrera, M., Pacheco, P., Palomar, J. y Zavala, D. (2010). La adicción a Facebook relacionada con la baja autoestima, la depresión y la falta de habilidades sociales. Psicología Iberoamericana, 18(1), 6-18.

Instituto Nacional de Estadística. (2018). Encuesta sobre equipamiento y uso de tecnologías de información y comunicación en los hogares (año 2018). https://www.ine.es/prensa/ tich_2018.pdf

Jasso, J. L., López, F. y Díaz, R. (2017). Conducta adictiva a las redes sociales y su re- 
lación con el uso problemático del móvil. Acta de Investigación Psicológica, 7(3), 2.832-2.838. https://doi.org/10.1016/j.aipprr. 2017.11.001

Kampylis, P., Punie, Y. y Devine, J. (2015). A European framework for digitally-competent educational organisations. JRC Science for Policy Report. https://doi.org/10.2791/54070

Kim, S. Y., Kim, M. S., Park, B., Kim, J. H. y Choi, H. G. (2017). The associations between internet use time and school performance among Korean adolescents differ according to the purpose of internet use. PLOS ONE, 12(4). https://doi.org/10.1371/ journal.pone.0174878

Koyuncu, T., Unsal, A. y Arslantas, D. (2014). Assessment of internet addiction and loneliness in secondary and high school students. The Journal of the Pakistan Medical Association, 64(9), 998-1.002. https://pubmed.ncbi. nlm.nih.gov/25823176/

Kuss, D. J. y Griffiths, M. D. (2017). Social networking sites and addiction: ten lessons learned. International Journal of Environmental Research and Public Health, 14, 1-17. https:// doi.org/10.3390/ijerph14030311

Kuss, D. J., Griffiths, M. D. y Binder, J. F. (2013). Internet addiction in students: prevalence and risk factors. Computers in Human Behavior, 29(3), 959-966.

Labrador, F., Requesens, A. y Helguera, M. (2015). Guía para padres y educadores sobre el uso seguro de internet, móviles y videojuegos. Fundación Gaudium. https://ies pereda.es/ampa/docs/Guia.para.padres. y.educadores.sobre.el.uso.seguro.de.Inter net,.moviles.y.videojuegos.pdf

Lai, F. y Kwan, J. (2017). Socioeconomic influence on adolescent problematic internet use through school-related psychosocial factors and pattern of internet use. Computers in Human Behavior, 68, 121-136. https://doi. org/10.1016/j.chb.2016.11.021
Lee, E. B. (2015). Too much information: heavy smartphone and Facebook utilization by African American young adults. Journal of Black Studies, 46(1), 44-61. https://doi.org/10.1177 \%2F0021934714557034

Lee, Z. y Cheung, C. (2014). Problematic use of social networking sites: the role of selfesteem. International Journal of Business and Information, 9(2), 143-159. https://pdfs. semanticscholar.org/37cb/ca2ee1526bfe 276b52cd1c500186651f632c.pdf

Li, W., O'Brien, J. E., Snyder, S. M. y Howard, M. O. (2016). Diagnostic criteria for problematic internet use among $U$. S. university students: a mixed-methods evaluation. PLOS ONE, 11(1), e0145981. https://doi.org/10.1371/jo urnal.pone.0145981

Liu, Ch. y Mab, J. (2018). Development and validation of the Chinese social media addiction scale. Personality and Individual Differences, 134, 55-59. https://doi.org/10.1016/j.paid. 2018.05.046

Llamas, F. y Pagador, I. (2014). Estudio sobre las redes sociales y su implicación en la adolescencia. Enseñanza \& Teaching, 32(1), 43-57. http://dx.doi.org/10.14201/et20143214357

Lozano-Blasco, R. y Cortés-Pascual, A. (2020). Usos problemáticos de internet y depresión en adolescentes: meta-análisis. Comunicar, 63, 109-120. https://doi.org/10.3916/C63-2 020-10

Malak, M. Z., Khalifeh, A. H. y Shuhaiber, A. H. (2017). Prevalence of internet addiction and associated risk factors in Jordanian school students. Computers in Human Behavior, 70, 556-563. https://doi.org/10.1016/j.chb.2 017.01.011

Malo, S., Martín, M. ${ }^{a}$ M. y Viñas, F. (2018). Excessive use of social networks: psychosocial profile of Spanish adolescents. Comunicar, 26(56), 101-110. https://doi.org/10.3916/C 56-2018-10 
Manca, S. y Ranieri, M. ${ }^{a}$ (2016). Facebook and the others. Potentials and obstacles of social media for teaching in higher education. Computers \& Education, 95, 216-230. https://doi. org/10.1016/j.compedu.2016.01.012

Marín, V., Sampedro, B. y Muñoz, J. M. (2015). ¿Son adictos a las redes sociales los estudiantes universitarios? Revista Complutense de Educación, 26 (núm. especial), 233-251. https://doi.org/10.5209/rev_RCED.2015. v26.46659

Marín, V., Vega, E. y Passey, D. (2019). Determination of problematic use of social networks by university students. RIED. Revista Iberoamericana de Educación a Distancia, 22(2), 135-152. https://doi.org/10.5944/ried. 22.2.23289

Marino, C., Gini, G., Vieno, A. y Spada, M. M. (2018). A comprehensive meta-analysis on Problematic Facebook Use. Computers in Human Behavior, 83, 262-277. https://doi. org/10.1016/j.chb.2018.02.009

Martínez-Piñeiro, E., Vila, E. y Gewerc, A. (2018). El papel de la familia en la construcción de la competencia digital. Revista Ibérica de Sistemas e Tecnologias de Informação, 28, 1-13. https://doi.org/10.17013/risti.28.1-13

Mateus, J. C. y Hernández, W. (2019). Design, validation, and application of a questionnaire on media education for teachers in training. Journal of New Approaches in Educational Research, 8(1), 34-41. https://doi.org/10.782 1/naer.2019.1.329

Mayor, V., García, R. y Rebollo, Á. (2019). Explorando factores predictores de la competencia digital en las redes sociales virtuales. Píxel-Bit. Revista de Medios y Educación, 56, 51-69. https://doi.org/10.12795/pixelbit.2019.156.03

Mazman, S. G. y Usluel, Y. K. (2011). Modeling educational usage of Facebook. Computers \& Education, 55, 2, 444-453. https://doi.org/ 10.1016/j.compedu.2010.02.008
Ministerio de Educación y Formación Profesional. (2019). PISA 2018. Programa para la evaluación internacional de los estudiantes. Informe español.

Mora, F., Dolengevich, H. y Quintero, J. (2015). Protocolo de tratamiento de las adicciones sin sustancia psicoactiva. Medicine. Programa de Formación Médica Continuada Acreditado, 11(86), 5.157-5.160.

Moreno, N., González, A., Torres, A. y Araya, J. (2017). Alfabetización digital a padres de familia en el uso de las redes sociales. Alteridad. Revista de Educación, 12(1), 8-19. https:// doi.org/10.17163/alt.v12n1.2017.01

Müller, K., Dreier, M., Beutel, M., Duven, E., Giralt, S. y Wölfling, K. (2015). A hidden type of internet addiction? Intense and addictive use of social networking sites in adolescents. Computers in Human Behavior, 55, 172-177. https://doi.org/10.1016/j.chb.2015.09.007

Musiał, K. y Kazienko, P. (2013). Social networks on the internet. World Wide Web, 16(1), 31-72. https://doi.org/10.1007/s1128 0-011-0155-Z

ONTSI. (2019). Informe sobre la sociedad de la información y las telecomunicaciones y el sector TIC y de los contenidos en España por comunidades autónomas. Ministerio de Economía y Empresa. https://www.ontsi.red. es/sites/ontsi/files/2019-10/Informe\%20 Espa\%C3\%B1a.pdf

Oppenheimer, A. (2018). jSálvese quien pueda!: el futuro del trabajador en la era de la automatización. Debate.

Orange. (2018). Guía sobre el uso responsable de la tecnología en el entorno familiar.

Orosz, G., Tóth-Király, Y. y Bothe, B. (2016). Four facets of Facebook intensity-The development of the multidimensional Facebook intensity scale. Personality and Individual Differences, 100, 95-104. https://doi.org/10. 1016/j.paid.2015.11.038 
Özdemir, Y., Kuzucu, Y. y Ak, Ş. (2014). Depression, loneliness and internet addiction: how important is low self-control? Computers in Human Behavior, 34, 284-290. https://doi. org/10.1016/j.chb.2014.02.009

Peñalva, A. y Napal, M. ${ }^{a}$ (2019). Hábitos de uso de internet en niños y niñas de 8 a 12 años: un estudio descriptivo. Hamut'ay, 6(2), 55-68. https://doi.org/10.21503/hamu.v6i2. 1775

Pérez-Escoda, A., García-Ruiz, R. y Aguaded, I. (2019). Dimensions of digital literacy based on five models of development. Cultura $y$ Educación, 31(2), 232-266. https://doi.org/1 0.1080/11356405.2019.1603274

Plaza, J. (2018). Riesgos percibidos por estudiantes adolescentes en el uso de las nuevas tecnologías y cómo reaccionan ante ellos. Bordón, 70(2), 105-120. https://doi. org/10.13042/Bordon.2018.55486

Pontes, H., Szabo, A. y Griffiths, M. (2015). The impact of internet based specific activities on the perceptions of internet addiction, quality of life, and excessive usage: a crosssectional study. Addictive Behaviors Reports, 1, 19-25. https://doi.org/10.1016/j.abrep.20 15.03.002

Rad, M. y Ahmadi, F. (2018). A new method to measure and decrease the online social networking addiction. Asia-Pacific Psychiatry. https://doi.org/10.1111/appy.12330

Redecker, C. y Punie, Y. (2017). Digital Competence of Educators DigCompEdu. Publications Office of the European Union.

Reed, P. y Reay, E. (2015). Relationship between levels of problematic internet usage and motivation to study in university students. Higher Education, 70(4), 711-723. https://doi. org/10.1007/s10734-015-9862-1

Rooij, A. van y Prause, N. (2014). A critical review of internet addiction criteria with suggestions for the future. Journal of Behavioral Addic- tions, 3(4), 203-213. https://doi.org/10.1556/ JBA.3.2014.4.1

Rubio, I. (2 de abril de 2019). La «generación muda»: los jóvenes que apenas llaman por teléfono. El País. https://elpais.com/tecnolo gia/2019/04/02/actualidad/1554220116 _828140.html

Rücker, J., Akre, C., Berchtold, A. y Suris, J. C. (2015). Problematic internet use is associated with substance use in young adolescents. Acta Paediátrica, 104, 504-507. https:// doi.org/10.1111/apa.1297

Şahin, C. (2018). Social media addiction scaleStudent form: the reliability and validity study. TOJET. The Turkish Online Journal of Educational Technology, 17(1), 168-182.

Sánchez, L., Benito, R., Serrano, A., Aleixandre, R. y Bueno, F. J. (2018). Programa de prevención del uso problemático de internet y de las redes sociales "Clikeando". Ajuntament de València.

Sandoval-Ato, R., Vilela-Estrada, M. A., Mejía, C. R. y Caballero-Alvarado, J. (2018). Suicide risk associated with bullying and depression in high school. Revista Chilena de Pediatría, 89(2), 208-215. https://doi.org/10.4067/S03 70-41062018000200208

Sanz, C., Martínez, A., Ballester, M. ${ }^{2}$ L. y Domínguez, A. (2017). Instrumentos de evaluación del uso problemático del teléfono móvil/ smartphone. Health and Addictions, 17(1), 5-14.

Schou, C., Torsheim, T., Scott, G. y Pallesen, S. (2012). Development of a Facebook addiction scal. Psychological Reports, 110(2), 501-517.

Se-Hoon, J., HyoungJee, K., Jung-Yong, Y. y Yoori, H. (2016). What type of content are smartphone users addicted to?: SNS vs. games. Computers in Human Behavior, 54, 10-17. https://doi.org/10.1016/j.chb.2015. 07.035 
Sigerson, L. y Cheng, C. (2018). Scales for measuring user engagement with social network sites: a systematic review of psychometric properties. Computers in Human Behavior, 83, 87-105. https://doi.org/10.1016/j.chb. 2018.01.023

Syahputra, Y., Prayitno, P., Syahniar, S., Karnely, Y. y Hariyani, H. (2019). Rasch stacking analysis of student internet addiction based on gender. Journal Konseling dan Pendidikan, 7(1), 35-41. https://doi.org/10.29210/ 129300

Tang, J. H., Chen, M. C., Yang, C. Y., Chung, T. Y. y Lee, Y. A. (2016). Personality traits, interpersonal relationships, online social support, and Facebook addiction. Telematics and Informatics, 33(1), 102-108. https://doi.org/10.10 16/j.tele.2015.06.003

Tao, R., Huang, X., Wang, J., Zhang, H., Zhang, Y. y Li, M. (2010). Proposed diagnostic criteria for internet addiction. Addiction, 105(3), 556-564.

Tejada, E., Castaño, C. y Romero, A. (2019). Los hábitos de uso en las redes sociales de los preadolescentes. RIED. Revista Iberoamericana de Educación a Distancia, 22(2), 119-133. https://doi.org/10.5944/ried. 22.2.23245

Turel, O. y Serenko, A. (2012). The benefits and dangers of enjoyment with social networking websites. European Journal of Information Systems, 21, 512-528. https://doi.org/10.1 057/ejis.2012.1

Turel, O., He, Q., Xue, G., Xiao, L. y Bechara, A. (Diciembre 2014). Examination of neural systems sub-serving Facebook «addiction». Psychological Reports, 115(3), 675-695. https:// doi.org/10.2466/18.PR0.115c31z8

Tutgun-Ünal, A. y Deniz, L. (2015). Development of the social media addiction scale. AJIT-e: Online Academic Journal of Information Technology, 6(2)1, 51-70. https://doi.org/10. 5824/1309-1581.2015.4.004.x
UNESCO. (1996). La educación encierra un tesoro. Santilla-UNESCO.

UNESCO. (2013). Directrices para las políticas del aprendizaje móvil.

Valencia, R. y Castaño, C. (2019). Use and abuse of social media by adolescents: a study in Mexico. Píxel-Bit. Revista de Medios y Educación, 54, 7-28. https://doi.org/10.12 795/pixelbit.2019.i54.01

Valerio, G. y Serna, R. (2018). Redes sociales y bienestar psicológico del estudiante universitario. Revista Electrónica de Investigación Educativa, 20(3), 19. https://doi.org/10.2432 O/redie.2018.20.3.1796

Vázquez, E. y Sevillano-García, M. ${ }^{a}$ L. (2018). Ubiquitous educational use of mobile digital devices. A general and comparative study in Spanish and Latin America higher education. Journal of New Approaches in Educational Research, 7(2), 105-115. https://doi.org/10. 7821/naer.2018.7.308

Vizcaíno-Verdú, A., Contreras-Pulido, P. y Guzmán-Franco, M. ${ }^{a}$ D. (2020). Construcción del concepto fanbullying: revisión crítica del acoso en redes sociales. Píxel-Bit. Revista de Medios y Educación, 57, 211-230. https://doi. org/10.12795/pixelbit.2020.157.09

Wang, L., Luo, J., Bai, Y., Kong, J., Gao, W. y Sun, X. (2013). Internet addiction of adolescents in China: prevalence, predictors, and association with well-being. Addiction Research \& Theory, 21(1), 62-9. https://doi. org/10.3109/16066359.2012.690053

Watson, R. (2011). Mentes del futuro. Viceversa.

Watters, C. A., Keefer, K. V., Kloosterman, P. H., Summerfeldt, L. J. y Parker, J. D. A. (2013). Examining the structure of the internet addiction test in adolescents: a bifactor approach. Computers in Human Behavior, 29(6), 2.294-2.302. https://doi.org/10.1016/ j.chb.2013.05.020

Wegmann, E., Stodt, B. y Brand, M. (2015). Addictive use of social networking sites can 
be explained by the interaction of internet use expectancies, internet literacy, and psychopathological symptoms. Journal of Behavioral Addictions, 4(3), 155-162. https://doi.org/ 10.1556/2006.4.2015.021

Xanidis, N. y Brignell, C. M. (2016). The association between the use of social network sites, sleep quality and cognitive function during the day. Computers in Human Behavior, 55, 121-126. https://doi.org/10.1016/j.chb.2015. 09.004

Yeganehfar, M., Zarei, A., Isfandyari-Mogghadam, A. R. y Famil-Rouhani, A. (2018). Justice in technology policy: a systematic review of gender divide literature and the marginal contribution of women on ICT. Journal of Information, Communication and Ethics in
Society, 16(2), 123-137. https://doi.org/10.1 108/jices-06-2017-0038

Young, K. (1998). Internet addiction: diagnosis and treatment considerations. Journal of Contemporary Psychotherapy, 39(4), 241-246. https://doi:10.1007/s10879-009-9120-x

Young, K. (2015). The evolution of internet addiction disorder. En C. Montang y R. Martin. (Eds.), Internet Addiction, Neuroscientific Approaches and Therapeutical Interventions (pp. 3-17). Springer International Publishing.

Yu, S., Wu, A. M. S. y Pesigan, I. J. A. (2016). Cognitive and psychosocial health risk factors of social networking addiction. International Journal of Mental Health and Addiction, 14(4), 550-564. https://doi.org/10.1007/s11 469-015-9

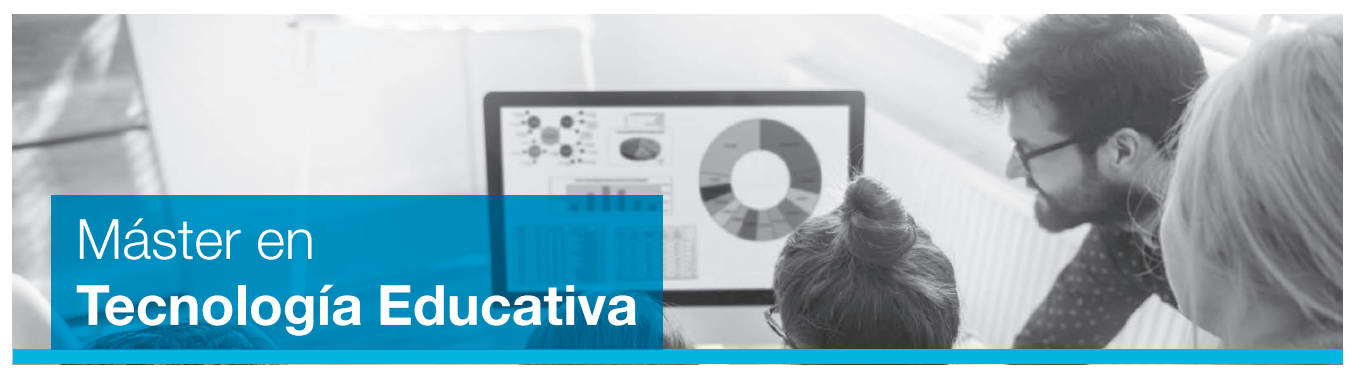

\section{Este máster oficial [60 créditos ECTS] tiene una duración normal de 12 meses.}

Dirigido a: Titulados universitarios de las distintas ramas del conocimiento que deseen especializarse en el correcto desempeño de las funciones de un experto en tecnología educativa. No exige experiencia previa en el ámbito educativo. Especialmente dirigido a titulados en Magisterio, Pedagogía y Educación Social.

Aquellas personas interesadas que no provengan de las titulaciones anteriormente citadas deberán realizar unos complementos formativos.

Objetivos: Capacitar a profesores, investigadores y educadores en el conocimiento y empleo de las nuevas tecnologías de la comunicación y la información, así como de los modelos formativos e-learning y b-learning, en beneficio de las acciones formativas en los nuevos contextos educativos. También profundiza en el conocimiento de las posibilidades que ofrecen las tecnologías actuales y emergentes para encontrar nuevas formas de obtención y manejo de información en ámbitos educativos. 


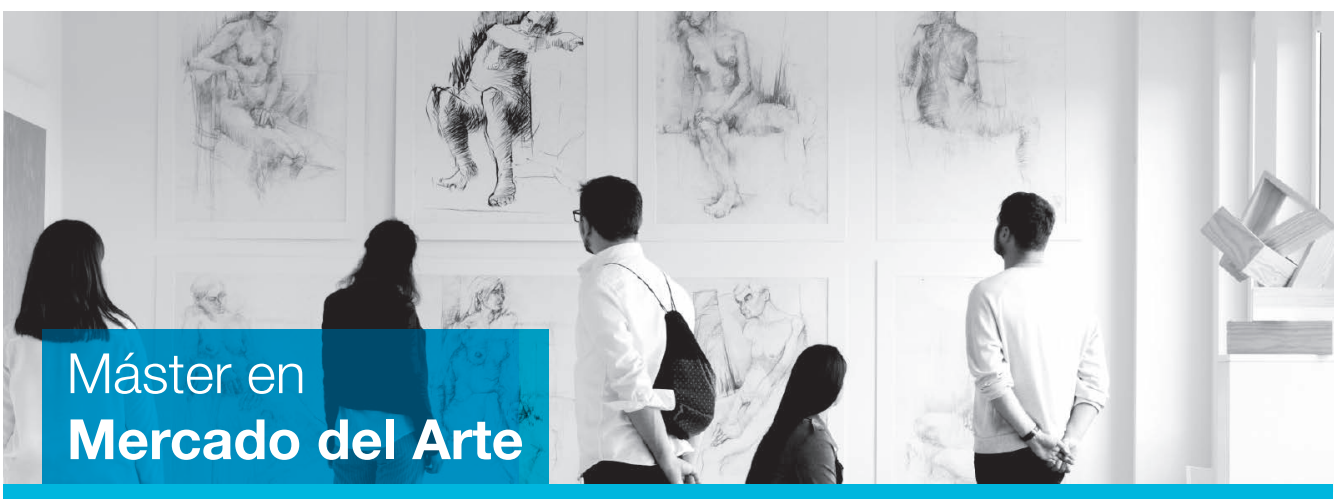

Este máster oficial de Mercado del Arte [60 créditos ECTS] tiene una duración normal de 12 meses.

Dirigido a: Titulados universitarios con un perfil amplio y diverso, que va desde el alumno con sesgo claramente humanista (como licenciados en Historia, Humanidades, Historia del Arte, Arqueología, etc.), hasta el alumno formado en finanzas, empresa y asesoramiento financiero que quiere encauzar sus conocimientos y experiencia hacia el mercado del arte y en el asesoramiento patrimonial en este tipo de activos. Tienen acceso directo al máster los titulados universitarios en Ciencias Empresariales, Administración y Dirección de Empresas y Economía. Aquellos titulados que no provengan de las titulaciones antes citadas deberán realizar unos complementos formativos.

Objetivos: Formar profesionales altamente cualificados en el ámbito de la dirección y gestión de empresas culturales relacionadas con el sector, dotándolos de un perfil práctico, profesional y competitivo. El programa formativo de este máster ha sido diseñado para que el estudiante domine todos los conocimientos necesarios que le capaciten para realizar adecuadamente y con garantía de éxito una labor de gestión, asesoramiento, peritaje y catalogación de bienes artísticos y de colección en entornos nacionales e internacionales.

\section{Publicaciones de interés}

\section{Área de Tecnología, Ciencia y Educación}

\section{Historia del pensamiento contemporáneo}

Juan Padilla Moreno

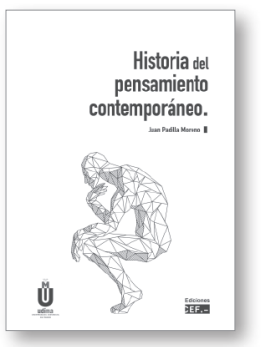

El objeto de este manual es la evolución del pensamiento en los dos últimos siglos de la historia occidental, desde finales del siglo XVIII hasta la actualidad. Siendo el tramo más reciente de una historia milenaria iniciada en la Grecia antigua, es también el más denso y complicado. A dos épocas claramente diferenciadas en el siglo XIX, el Romanticismo y la época positivista, sucede una gran crisis a comienzos del XX, que afecta no solo a la filosofía, sino también a la ciencia, la política, el arte o la religión. Desde entonces la historia del pensamiento se hace más fragmentaria y tanto más problemática cuanto más se aproxima a la actualidad.

En esta obra, el autor prima ante todo la claridad, especialmente necesaria por tratarse de un pensamiento que, dada su larga historia, se ha hecho sumamente sutil y complejo, pero procurando ser riguroso y fiel a las fuentes. 